\title{
Investigation and Cultivation Strategies of Chinese Character Writing Ability of Students in Normal Universities Against the Background of Informationization*
}

\author{
Juan Liu \\ Heihe University \\ Heihe, China 164300
}

\begin{abstract}
Chinese character writing ability is an important part of humanistic quality of college students, and also an educational and teaching ability that normal students should be equipped as a people's teacher in the future. In view of the investigation, there are some problems in Chinese character writing ability of normal students. Therefore, colleges and universities must attach importance to the education of standardized Chinese character writing.
\end{abstract}

Keywords-normal student; Chinese character writing ability; survey; cultivation strategies

\section{INTRODUCTION}

The advent of the information age has had a great impact on the writing of Chinese characters. The keyboard gradually replaces the writing tools, and the input method supersedes the writing process, and the standardized Chinese character writing is gradually neglected by people. The standardized Chinese character writing is an important standard for professional skills of normal students. The standardized Chinese character writing ability is an indispensable skill for normal students to step onto the platform. In recent years, the writing level of standardized Chinese characters of normal students has declined. How to improve normal students' writing level of Chinese characters under the background of informationization is a problem demanding prompt solution. This paper combines the available relational approaches in the information age with the teaching of standardized writing of Chinese characters, and strives to provide some strategies and ways for normal students' standardized writing of Chinese characters under the background of informationization.

II. INVESTIGATION AND ANALYSIS OF THE STATUS QUO OF CHINESE CHARACTER WRITING ABILITY OF NORMAL STUDENTS

As the Internet era is coming, people overly rely on computer and gradually alienate from writing of Chinese characters. "Character amnesia", "poor handwriting", and "incapable of writing" have become a commonplace matter. The writing level of Chinese characters of some college

*College Students' Innovation and Entrepreneurship Training Program in Heilongjiang Province (No.: 201713744037). students is even more worrying. As normal students majoring in normal education in colleges and universities, they should not only have extensive professional knowledge and ability to impart knowledge, but also have strong Chinese character writing ability. Teachers' correct and standardized literal expression is an important means to achieve a good educational effect. Good handwriting is an indispensable and basic professional skill for teachers. Therefore, as normal students, their ability to write Chinese characters is very important.

With the theme of "Chinese character writing ability of normal students", in May 2016, the author randomly selected 500 normal education students for questionnaires and interviews in one undergraduate normal university and two comprehensive ordinary universities respectively, and learned about the Chinese character writing ability of some normal students.

\section{A. The Status of the Questionnaire Survey}

1) The phenomenon of character amnesia: Through the analysis of the questionnaire, nearly three-quarters of the normal students have admitted that there is a phenomenon of character amnesia. When encountering this situation, only about one-third of students have said they choose to make inquiries by means of tools, and most of them choose to ask others, and a few students choose to use other words to replace the required one.

2) The phenomenon of wrongly written or mispronounced characters: Wrongly written or mispronounced characters are a common problem existing in college students. Some students make mistakes because some Chinese characters have the same or similar pronunciation, and the other reason is that some Chinese characters have similar character pattern. The former accounts for a larger proportion. More than onehalf of students believe that wrongly written or mispronounced characters must be corrected, and a few believe that wrongly written or mispronounced characters may not be corrected as long as they do not affect communication and understanding. 
3) The phenomenon of wrong stroke order: It can be seen from the survey that students pay less attention to stroke order. Only one in five students notice stroke order when they are writing. Most students pay special attention to strokes when they encounter special Chinese characters. A few students believe that Chinese characters have been written correctly, and it doesn't matter whether stroke order is correct or not.

4) The phenomenon of writing: According to the survey, only one in five students write frequently, and one in three students basically do not write except when necessary (such as the exam), and one half of the students take notes in class. Apart from the handwriting required by the teachers, students will choose to complete the task on a computer. Most people choose arithmetic of smart spell input method, which also reduces the students' understanding and memory of Chinese characters.

Based on the above findings, the phenomenon of normal students' character amnesia occurs most frequently, which is a common and universal problem. Next, the phenomenon of wrongly written or mispronounced characters is also a very common problem. Although the stroke order error is not a very common problem, it has very high frequency of occurrence.

\section{B. Analysis of Influencing Factors}

According to the findings, the main factors that influence normal students' Chinese character writing ability are:

The first is the self-recognition and degree of emphasis .Normal universities or normal education majors offer courses such as calligraphy, Three Calligraphies, etc. but students seldom practice actively after class. Some students cannot read a book seriously, resulting in a reduction in the accumulation of vocabulary. The more time students spend on computer, the less time they spend in traditional writing. They also use a large number of network symbols to express their meaning. The development of these habits will gradually reduce their ability to write Chinese characters.

The second is the writing frequency. The lower frequency of writing is, the more problems exist in writing. Those students who often keep diaries, take notes and write compositions will have a better ability of Chinese character writing than those who often use computers to type without writing.

The third is network environment. The computer has a great impact on students' Chinese character writing ability. They believe that writing is not as fast as typing. They rely on computers and become lazy in Chinese character writing. The rapid spread of online buzzwords also affects students' correct understanding of Chinese characters and words to a certain extent.

The fourth is campus environment. The demand for the education of Chinese character writing in colleges and universities is generally lower than that of professional education. Many of the teaching links are gradually completed by computer, and all kinds of notifications and propaganda are mostly completed by printing, rarely in the form of handwriting. School is the main place for the education of
Chinese character writing, and the creation of environment and atmosphere is of great importance.

The fifth is multimedia teaching means. Multimedia teaching is the preferred form for university teachers to teach. Few teachers want to influence students through standard and neat blackboard-writing. Teachers' role in demonstrating Chinese characters writing is reduced.

\section{THE IMPORTANCE AND CULTIVATION STRATEGIES OF IMPROVING NORMAL STUDENTS' CHINESE CHARACTER WRITING ABILITY}

\section{A. The Importance of Improving Normal Students' Chinese Character Writing Ability}

In the teaching process of professional knowledge education for normal students, colleges and universities should also strengthen the education and teaching in their Chinese writing ability, which not only is conductive to cultivating their aesthetic taste, but also improving their moral and cultural qualities.

1) Contributing to competence for future education teaching: The training object of normal education major in colleges and universities is the future primary and secondary school teachers, who bear the important responsibility of teaching and educating people. As people's teachers in the future, first of all, normal students must understand the state's requirements for the writing of Chinese characters, implement relevant state regulations, carefully study textbooks, and complete teaching tasks in accordance with state regulations. Second, they must increasingly pay attention to the writing requirements of Chinese characters, improve their Chinese character writing ability, write standardized Chinese characters, and avoid wrongly written or mispronounced characters and character amnesia. Third, they must know why, trace the source of wrong writing, find out the problem, and avert the same mistakes. Fourth, in the future teaching process, they must guide students to correctly understand the network and advise students not to use non-standard online buzzwords.

Therefore, for normal students, to develop good writing habits and improve their Chinese character writing ability plays a vital role in future education and teaching.

2) Helping to understand the essence of Chinese traditional culture: Chinese characters are not only the writing symbols of recording Chinese, but also the projection of colorful society. Every Chinese character is like a picture or a poem. As long as we truly master the Chinese characters, we can grasp the historical wisdom contained in them and appreciate their extraordinary charm. It is precisely because people can comprehend Chinese traditional culture through Chinese characters, so Chinese characters are called the gene of Chinese culture and the essence of Chinese traditional culture. In 2017, Chinese and Chinese characters were placed in the first place in Chinese Textbook of Compulsory Education Compiled by the Ministry of Education, which reflected the importance the country attached to in Chinese character learning and Chinese traditional culture. The 
purpose of the education of Chinese character writing to normal students is to make them deeply make out the connotation and essence of Chinese traditional culture, so as to consciously inherit Chinese traditional culture.

\section{B. Cultivation Strategies of Improving Normal Students' Chinese Character Writing Ability}

Through the above analysis, we can see that there are some problems in the writing of Chinese characters for normal students. It is very necessary to improve their Chinese characters writing ability.

1) Increasing chances of writing Chinese characters: We can enhance students' awareness of writing Chinese characters through activities related to Chinese characters. First, the professional assignment assigned by teachers can be completed by hands. Second, we can cultivate students' interest in calligraphy, connect the students' motivation to learn calligraphy with the students' academics, offer elective courses, hold calligraphy competitions, and organize calligraphy classes.

2) Developing good reading habits: The level of Chinese character recognizing and memorizing of normal students can be improved by promoting them to develop good reading habits. The firs is to provide a list of bibliographies related to traditional culture. The second is to create a atmosphere of "reading loudly". The third is to ask students to take reading notes carefully. The fourth is to hold a regular reading report to check the reading effect. These practices can not only improve the level of students' application of Chinese characters, but also enhance their cultural literacy.

3) Strengthening teachers' exemplary role: Teaching by deeds is more important than by words. Teachers should also lay emphasis on improvement of their writing quality. Teachers must strive to be correct, standardized and beautiful both in classroom blackboard-writing and after-class assignments correction, and truly play a role in leading and demonstration, so that students are imperceptibly affected. This may also be the one way to improve students' Chinese character writing ability.

4) Perfecting evaluation and assessment mechanism: Some colleges and universities have tested normal students' pen handwriting, chalk handwriting and brush handwriting in order to promote the improvement of their abilities in using the Chinese language. However, due to the imperfection of the assessment system and evaluation mechanism, students cannot attach great importance to it, which will ultimately affect their ability to write Chinese characters.

\section{CONCLUSION}

Fundamentally speaking, to improve normal students' Chinese character writing ability is to enhance their professional quality, which will ultimately greatly benefit them. Moreover, Chinese characters are an important carrier of Chinese culture and national spirit. The education of Chinese character writing is an important way to inherit traditional Chinese culture. College students should write Chinese characters in an accurate, standardized and artistic way, so as to pass on the long-standing Chinese character culture of the Chinese nation.

\section{REFERENCES}

[1] Dai Zhujun. The Social Significance and Strategy of Improving Students' Chinese Character Writing Ability in Normal Universities [J]. Journal of Educational Institute of Jilin Province, 2015, 31 (12): 102$103+115$.

[2] Ding Qinggang. Analysis of College Students' Chinese Character Writing Ability and Its Influencing Factors [J]. Modern Chinese, 2015 (11): 81-83

[3] Jia Min. Research on Contemporary College Students' W Chinese Character Writing Ability [D]. Shanxi Normal University, 2015. 